\title{
Impaired Cognitive Processes Influence Expressive Language Skills In Attention Deficits
}

\author{
Callyn Villanueva $\ddagger$ \\ ‡ Hofstra University, Hempstead, United States of America
}

\begin{abstract}
This research idea investigates neural interactions by utilizing transcranial magnetic stimulation (TMS) to disrupt neural activities between the frontal and temporal left hemisphere cortices in individuals when performing language related task. The initial purpose is to thoroughly examine how impaired cognitive processes alter expressive language skills in attention deficit disorder and create an experimental design suited for examination.
\end{abstract}

\section{Keywords}

neuroscience, attention, cognition

\section{Background}

Individuals who are diagnosed with Attention Deficit Hyperactivity Disorder have impaired executive functions that are critical for regulating cognitive processes. This functioning enables individuals to establish concept formations, have intact working memory, attention and expressive language. Understanding the essential biological mechanisms underlying cognitive process is the key to examining attention deficits. Previous neuro-cognitive 
studies elucidated executive dysfunction in cognitive deficiencies in ADHD. With the impairment of executive function, subjects often show various dependent cognitive deficits such as language and visuospatial impairments. Children with ADHD also show low verbal memory performance, poor inner speech, difficulty in concept formation and poor categorical reasoning in both visual and verbal tasks (Machinskaya and Semenova 2004).

\section{Literature Review}

A study by Krain and Castellanos (2006) explains the underlying physiological mechanisms associated with cognitive deficits using the pre-frontal striatal model. The model explains that changes in the pre-frontal cortex and stratiopallidal system influence inhibitory control in ADHD subjects. Researchers examined the relationships between regional brain volumes in those with ADHD using magnetic resonance imaging. The results found that there was a decrease in frontal lobe volumes with subjects who have ADHD. In contrast to this theory, other recent functional neuroimaging studies have revealed that cognitive deficits that are associated with ADHD may involve a variety of brain impairments. These studies have shown that there is lower activation of neural networks across brain regions in those with attention deficits compared to the control group during a cognitive task \& resting state (Castellanos and Proal 2012), involving the anterior medial PFC (aMPFC) and posterior cingulate cortex (PCC). The results show that these regions were diminished during cognitive task. The analysis of these regions could be further examined in a manner that would support whether cognitive processes in certain regions associate with specific cognitive activities; leading towards Machinskya's initial research. Machinskaya et al. (2014) examined Luria's approach Luria (1973)of the dynamic localization of higher mental functions to understand the interrelations between learning difficulties in regards to a specific functional system in the brain. Luria's theory describes that learning difficulties are caused by a lack of active components of mental function (ex. expressive language, planning, etc.) and with a lack of activation or maturity of a specific functional brain system associated with cognitive deficiencies. A structural EEG and frontal theta wave (FTW) analysis identified impairment in children with $A D H D$ in the fronto-thalamic region. The function of speech is provided by regions in the left hemisphere.

The impairment of speech could possibly be caused by the disturbance of both the left frontal \& temporal regions which connects to Machinskya's results of decreased cortical activity in the fronto-thalamic region; revealing that there is a decreased interaction between frontal and temporal left hemisphere cortices in subjects with ADHD; causing a dysfunction in verbal performance. A possibility for researchers to consider is to further analyze Machinskya's research in examining cortical interaction between frontal and temporal left hemisphere cortices by conducting a TMS to analyze whether the interaction between frontal \& temporal cortices impact expressive language skills (specifically in verbal communication skills). Verbal communication plays a vital role in social interactions. Because ADHD causes impaired cognitive functioning, children with ADHD who have difficulty with verbal expression also experience significant impairment in social functioning. Impairment of non-verbal working memory and fluency of language can cause a 
dysfunction in socialization and adaptive behavior in children (including adolescence) (Shoulberg 2011).

Do executive deficits alone address a dysfunction in expressive communication in social interaction? Bunford et al. (2015) recent study reveals that deficits of inhibition and working memory are heavily associated with social impairment of children by analyzing response inhibition and social interaction in an academic setting. The properties of speech in verbal communication are important aspects to social interaction. The properties of speech, examined in Green et al. (2014) research, demonstrates the difficulties in the pragmatic aspects of language in children with ADHD, such as excessive verbal speech \& interruption in communication, by conducting observational studies of children's communication patterns and studies of complex language comprehension and production. Identifying pragmatic components is essential for expressive language skills in that it refers to the appropriate and effective use of language in interpersonal contexts and is important for children's ability to function well at different social settings (Russell 2007). There are several explanations that have been proposed for language comprehension and production difficulties of children with ADHD. Regarding language production, Engelhardt et al. (2013) proposed that a deficit in response inhibition cause adult \& children to speak without planning what they have to say. In his study, he examines speech production by asking subjects to construct sentences using objects as verbs. His results show, for both children and adults, that there was a dysfunction in grammaticism and speech disfluency with one particular type of verb. In contrast, Purvis and Tannock (1997) suggest that monitoring information required for speech production requires a range of various executive functions, including working memory and control of attention; similar to Green's study which has shown similar results from previous studies in which executive function contributes to pragmatic language competency. Along with verbal language; written expression has also been studied in subjects with ADHD. DeBono et al. (2012) examined the interrelations between standardized measures of written expression and cognitive processing measures (working memory, processing speed, language, etc.). Consistent with previous studies, these executive functioning skills are critical for overall verbal and written expressive language. Impaired cognitive processes in attention deficits have a significant impact on expressive language skills.

The aforementioned studies have examined the underlying neural mechanisms \& cognitive processes that influence language skills in subjects with attention deficits. The regulation of executive functioning is essential for efficient verbal \& written communication. Impairment in this functioning will alter processes that contribute to expressive language skills, impacting the individual's ability to express in a language based manner.

\section{Research Question I}

Does the disruption of neural activities between the frontal and temporal left hemisphere cortices affect verbal expression? 


\section{Methodology I}

This experiment will consist of a 15 minute session of implementing transcranial magnetic stimulation (TMS) to the experimental group (10 participants with sufficient verbal language expression who are not diagnosed with any language/attention related disorders). TMS serves as a high frequency voltage towards specific neurons, creating a GABAergic inhibition. This inhibition disrupts cognitive activities in a brain region. The TMS will be located at the frontal \& temporal lobe.

After implementing TMS, the investigator will be examining aspects of verbal language using The Interagency Language Roundtable scale to both experimental \& control groups (normal subjects who have not been exposed to TMS \& to subjects with attention deficits). This examines language proficiency on a scale of 0-5 based on open ended questions. During this face to face interview, each question will be measured to assist in identifying whether the individuals meet a certain criteria in their verbal language skills. Conducting a comparison, TMS subjects would have scores similar or close to subjects with attention deficits while subjects who were not exposed to TMS would have a higher score range.

\section{Research Question II}

Does the disruption of neural activities between the frontal and temporal left hemisphere cortices affect written expression?

\section{Methodology II}

After implementing TMS (another 15 minute session), this experiment will replicate one of DeBono et al. (2012)methods of measuring written expression. Participants (both in experimental \& control groups) will construct a written narrative based on a stimulus picture within a time limit, in order to measure their writing abilities. The participants will be measured by The Towl-3 Spontaneous Writing Format, which is used to assess the ability to write an essay based on a presented picture within a certain time limit. This test utilizes 3 types of standardized scores for written expression performance including:

- Contextual Conventions (showing the accuracy of capitalization, spelling, and punctuation)

- $\quad$ Contextual Language (grammar, sentence construction \& ones vocabulary

- $\quad$ Story Construction (the description of elements)

Similar to the experiment analyzing verbal expression in conducting a comparison, TMS subjects would have scores similar or close to subjects with attention deficits while subjects who were not exposed to TMS would have a higher score range. 


\section{References}

- $\quad$ Bunford N, Brandt NE, Golden C, Dykstra JB, Suhr JA, Owens JS (2015) Attentiondeficit/hyperactivity disorder symptoms mediate the association between deficits in executive functioning and social impairment in children. Journal Of Abnormal Child Psychology 43 (1): 133-147. https://doi.org/10.1007/s10802-014-9902-9

- $\quad$ Castellanos FX, Proal E (2012) Large-scale brain systems in ADHD: beyond the prefrontal-striatal model. Trends in Cognitive Sciences 16 (1): 17-26. https:// doi.org/10.1016/i.tics.2011.11.007

- DeBono T, Hosseini A, Cairo C, Ghelani K, Tannock R, Toplak ME (2012) Written expression performance in adolescents with attention-deficit/hyperactivity disorder (ADHD). Reading and Writing 25 (6): 1403-1426. https://doi.org/10.1007/ s11145-011-9325-8

- $\quad$ Engelhardt P, Nigg J, Ferreira F (2013) Is the fluency of language outputs related to individual differences in intelligence and executive function? Acta Psychologica 144 (2): 424-432. https://doi.org/10.1016/j.actpsy.2013.08.002

- Green BC, Johnson KA, Bretherton L (2014) Pragmatic language difficulties in children with hyperactivity and attention problems: An integrated review. International Journal Of Language \& Communication Disorders 49 (1): 15-29. https:// doi.org/10.1111/1460-6984.12056

- Krain AL, Castellanos F (2006) Brain development and ADHD. Clinical Psychology Review 26 (4): 433-444. https://doi.org/10.1016/i.cpr.2006.01.005

- Luria AR (1973) The working brain: an introduction to neuropsychology. Basic Books, New York.

- Machinskaya R, Semenova O (2004) Peculiarities of formation of the cognitive functions in junior school children with different maturity of regulatory brain systems. Journal of Evolutionary Biochemistry and Physiology 40 (5): 528-538. https://doi.org/10.1007/ s10893-005-0009-3

- $\quad$ Machinskaya RI, Semenova OA, Absatova KA, Sugrobova GA (2014) Neurophysiological factors associated with cognitive deficits in children with ADHD symptoms: EEG and neuropsychological analysis. Psychology \& Neuroscience 7 (4): 461-473. https://doi.org/10.3922/i.psns.2014.4.05

- $\quad$ Purvis KL, Tannock R (1997) Language abilities in children with ADHD, reading disabilities and normal controls. Journal of Abnormal Child Psychology 25: 133-144. https://doi.org/10.1023/A:1025731529006

- Russell RL (2007) Social communication impairments: pragmatics. Pediatric Clinics of North America 54 (3): 483-506. https://doi.org/10.1016/i.pcl.2007.02.016

- Shoulberg H (2011) Activity intervention reduces severity of ADHD symptoms in young children. Journal of Attention Disorders 17 (1): 70-82. https:// doi.org/10.1177/1087054711417395 